ARTICLE

https://doi.org/10.1038/s41467-019-10588-0

\title{
Hypoxia-enhanced Blood-Brain Barrier Chip recapitulates human barrier function and shuttling of drugs and antibodies
}

\author{
Tae-Eun Park (1) 1,6,9, Nur Mustafaoglu1,9, Anna Herland 1,7,8, Ryan Hasselkus (1) 1, Robert Mannix 1,5, \\ Edward A. FitzGerald (10) ${ }^{1}$, Rachelle Prantil-Baun ${ }^{1}$, Alexander Watters ${ }^{1}$, Olivier Henry ${ }^{1}$, Maximilian Benz (1) 1, \\ Henry Sanchez ${ }^{1}$, Heather J. McCrea², Liliana Christova Goumnerova (iD ${ }^{2}$, Hannah W. Song ${ }^{3}$, Sean P. Palecek ${ }^{3}$, \\ Eric Shusta ${ }^{3} \&$ Donald E. Ingber (1) 1,4,5
}

The high selectivity of the human blood-brain barrier (BBB) restricts delivery of many pharmaceuticals and therapeutic antibodies to the central nervous system. Here, we describe an in vitro microfluidic organ-on-a-chip BBB model lined by induced pluripotent stem cellderived human brain microvascular endothelium interfaced with primary human brain astrocytes and pericytes that recapitulates the high level of barrier function of the in vivo human BBB for at least one week in culture. The endothelium expresses high levels of tight junction proteins and functional efflux pumps, and it displays selective transcytosis of peptides and antibodies previously observed in vivo. Increased barrier functionality was accomplished using a developmentally-inspired induction protocol that includes a period of differentiation under hypoxic conditions. This enhanced BBB Chip may therefore represent a new in vitro tool for development and validation of delivery systems that transport drugs and therapeutic antibodies across the human BBB.

\footnotetext{
${ }^{1}$ Wyss Institute for Biologically Inspired Engineering at Harvard University, Boston, MA 02115, USA. ${ }^{2}$ Department of Neurosurgery, Boston Children's Hospital and Harvard Medical School, Boston, MA 02115, USA. ${ }^{3}$ Department of Chemical and Biological Engineering, University of Wisconsin-Madison, Madison, WI 53706, USA. ${ }^{4}$ Harvard John A. Paulson School of Engineering and Applied Sciences, Harvard University, Cambridge, MA 02138, USA

${ }^{5}$ Vascular Biology Program and Department of Surgery, Boston Children's Hospital and Harvard Medical School, Boston, MA 02115, USA. ${ }^{6}$ Present address: Ulsan National Institute of Science and Technology (UNIST), UNIST-gil 50, Ulsan 44919, Republic of Korea. ${ }^{7}$ Present address: Division of Micro and Nanosystems, KTH Royal Institute of Technology, Stockholm, Sweden. ${ }^{8}$ Present address: Swedish Medical Nanoscience Center, Department of Neuroscience, Karolinska Institute, Stockholm, Sweden. ${ }^{9}$ These authors contributed equally: Tae-Eun Park, Nur Mustafaoglu. Correspondence and requests for materials should be addressed to D.E.I. (email: don.ingber@wyss.harvard.edu)
} 
T he human blood-brain barrier (BBB) is a unique and selective physiological barrier that controls transport between the blood and the central nervous system (CNS) to maintain homeostasis for optimal brain function. The BBB is composed of brain microvascular endothelial cells (BMVECs) that line the capillaries as well as surrounding extracellular matrix (ECM), pericytes, and astrocytes, which create a microenvironment that is crucial to BBB function ${ }^{1}$. The brain microvascular endothelium differs from that found in peripheral capillaries based on its complex tight junctions, which restrict paracellular transit and instead, require that transcytosis be used to transport molecules from the blood through the endothelium and into the CNS $^{2}$. BMVECs also express multiple broad-spectrum efflux pumps on their luminal surface that inhibit uptake of lipophilic molecules, including many drugs, into the brain ${ }^{3,4}$. The astrocytes and pericytes provide signals that are required for differentiation of the BMVECs ${ }^{5,6}$, and all three cell types are needed to maintain $\mathrm{BBB}$ integrity in vivo as well as in vitro ${ }^{7,8}$. The $\mathrm{BBB}$ is also of major clinical relevance because dysfunction of the $\mathrm{BBB}$ is observed in many neurological diseases, and the efficacy of drugs designed to treat neurological disorders is often limited by their inability to cross the $\mathrm{BBB}^{9}$. Unfortunately, neither animal models of the BBB nor in vitro cultures of primary or immortalized human BMVECs alone effectively mimic the barrier and transporter functions of the BBB observed in humans ${ }^{10-12}$. Thus, there is a great need for a human BBB model that could be used to develop new and more effective CNS-targeting therapeutics and delivery technologies as well as advance fundamental and translational research 78 .

Development of human induced pluripotent stem (iPS) cell technology has enabled differentiation of brain-like microvascular endothelial cells (iPS-BMVECs) that exhibit many properties of the human $\mathrm{BBB}$, including well-organized tight junctions, expression of nutrient transporters and polarized efflux transporter activity ${ }^{13,14}$. The trans-endothelial electrical resistance (TEER) values exhibited by the permeability barrier generated by these human iPS-BMVECs reach physiological levels $\left(\sim 3000-5000 \Omega \cdot \mathrm{cm}^{2}\right)$ within $24-48 \mathrm{~h}$ when cultured in Transwell inserts or within a microfluidic organ-on-a-chip (Organ Chip) device $^{15-17}$, a level that is more than an order of magnitude higher than TEER values previously reported in other in vitro human BBB models 6,15 . However, the usefulness of these iPSBMVEC models for studies on targeted delivery to the CNS is limited because they can only maintain these high TEER levels for $\sim 2$ days, and the expression of efflux pumps in these iPSBMVECs does not fully mimic those of human brain endothelium in vivo ${ }^{18}$. Here, we describe the development of an enhanced human BBB model created with microfluidic Organ Chip culture technology ${ }^{19}$ that contains human iPS-BMVECs interfaced with primary human pericytes and astrocytes, and that uses a developmentally inspired differentiation protocol ${ }^{20-22}$. The resulting human BBB Chip exhibits physiologically relevant levels of human BBB function for at least 1 week in vitro, including low barrier permeability and expression of multiple efflux pumps and transporter functions that are required for analysis of drug and therapeutic antibody transport.

\section{Results}

Developmentally inspired differentiation of brain endothelium. Given that the BBB first forms in the developing brain in a relatively oxygen-poor environment (1-8\%) before establishment of the circulatory system ${ }^{23}$, and oxygen availability has been shown to play a vital role in endothelial differentiation from a variety of stem cell sources ${ }^{20,21}$, we hypothesized that culturing iPS cells under similar hypoxic conditions could generate more highly differentiated BMVECs and potentially stabilize their phenotype. The published differentiation induction protocol for creating human BMVECs involves culture of iPS cells on Matrigel for 3 days in mTeSR1 medium followed by 6 days in DMEM-F12 medium, and then 3 days in endothelial cell medium containing retinoic acid under normoxic $\left(20 \% \mathrm{O}_{2}\right)$ conditions $^{13}$. Thus, to explore this possibility, we modified this method for inducing differentiation of BMVECs from human iPS cells by shifting the cultures to hypoxic conditions $\left(5 \% \mathrm{O}_{2}\right)$ for the last 9 days of the induction protocol (Supplementary Figure 1a). Importantly, we found that exposure to hypoxia during this differentiation protocol produced significant (2-6-fold) increases in the mRNA levels for the endothelial cell-cell adhesion molecules, VEcadherin (vascular endothelial cadherin) and PECAM-1 (platelet endothelial cell adhesion molecule, also known as CD31), as well as the influx transporter GLUT-1 (BBB-specific glucose transporter), efflux transporter P-gp (permeability glycoprotein), and VEGF-A (angiogenic vascular endothelial growth factor-A), relative to control iPS-BMVECs induced under normoxic conditions (Supplementary Figure 1b and Supplementary Table 1). The increased expression of these endothelial molecules that play an essential role in CNS neovascularization ${ }^{24,25}$ confirmed the successful transition of the iPS cells into a human BMVEC phenotype.

Canonical Wnt/ $\beta$-catenin signaling is essential for development of brain microvessels, and Wnt ligands Wnt7a and Wnt7b have been implicated in BBB development in vivo ${ }^{16,26}$. We similarly found that Wnt7a mRNA levels increased by over 25 -fold with the hypoxic induction protocol compared with normoxic conditions, while Wnt7b levels remained unchanged (Supplementary Figure $1 \mathrm{c}$ and Supplementary Table 2). In addition, past in vitro and in vivo studies showed that $\mathrm{Wnt} / \beta$-catenin signaling pathways interplay with hypoxia-induced factor $1 \alpha$ (HIF1a) signaling 27,28 . Indeed, ELISA analysis revealed that HIFla protein levels were upregulated by exposure to hypoxia during the differentiation protocol; however, they decreased when the differentiation was completed and the cells shifted to normoxic conditions after seeding on-chip (Supplementary Figure 1d). Interestingly, we found that we could produce similar results by exposing the iPS cells to $100 \mu \mathrm{M}$ cobalt chloride $\left(\mathrm{CoCl}_{2}\right)$ for the same 9- day period while under normoxic conditions (Supplementary Figure 1d); $\mathrm{CoCl}_{2}$ has been previously shown to chemically mimic the effects of hypoxia by stabilizing HIF $1 \alpha^{29}$. This finding may obviate the need for specialized gases and culture chambers for induction of improved BMVEC differentiation in future studies.

Reconstitution of a human BBB on-chip under hypoxia. To explore whether these hypoxia-induced iPS-BMVECs could be used to build a human BBB Chip with enhanced functionality, we used soft lithography to create optically clear, poly(dimethylsiloxane) (PDMS), 2-channel, microfluidic devices containing an upper CNS microchannel separated from a parallel vascular microchannel by a porous $(2 \mu \mathrm{m}$ diameter $)$, polyethylene terephthalate (PET) membrane coated on both sides with an ECM composed of collagen type IV and fibronectin (Fig. 1a). A mixture of primary human brain astrocytes and pericytes (seeding ratio of 7:3) were then cultured in the astrocyte medium on the PET membrane in the upper channel under static conditions for $1 \mathrm{~h}$. Human iPS-BMVECs cultured for 8 days under hypoxic $\left(5 \% \mathrm{O}_{2}\right)$ conditions on Matrigel were removed using Accutase and plated on the bottom surface of the ECM-coated porous membrane in the lower channel of the microfluidic chip in endothelial medium with addition of retinoic acid (RA) using similar culture conditions and flow rate. Chips were flipped and incubated for $5 \mathrm{~h}$ to induce adhesion of iPS-BMVECs to the membrane. The BBB 

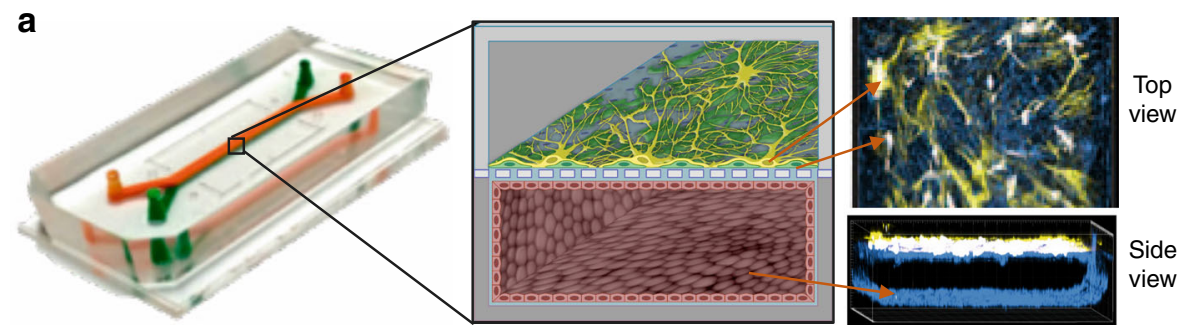

b

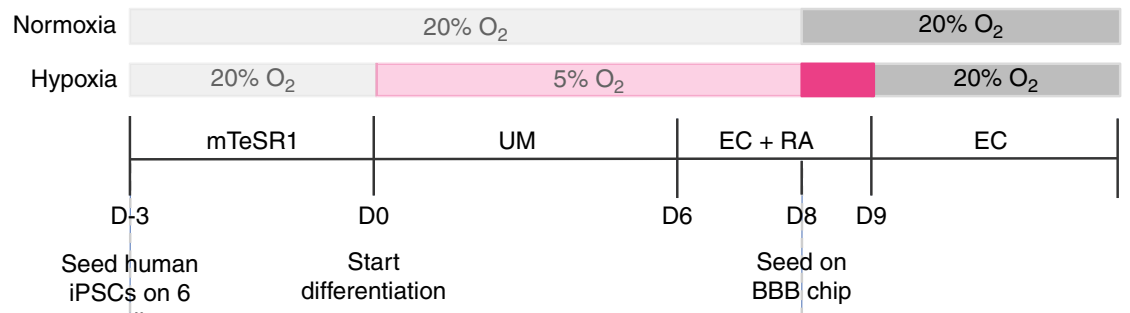

wells

On 6-well plate

C

ZO-1

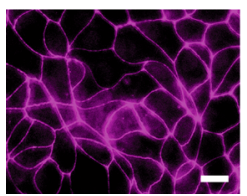

d
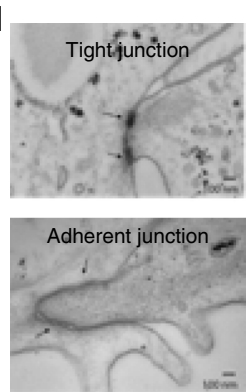

Claudin-5

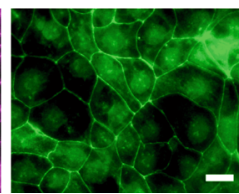

e

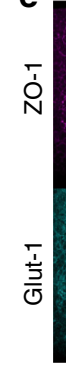

PECAM-1

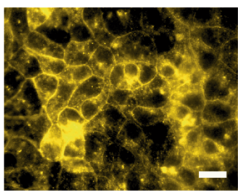

Glut-1

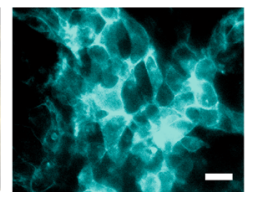

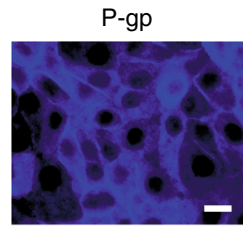

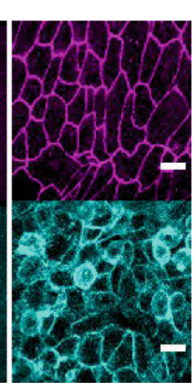

Fig. 1 Reconstitution of the human BBB in an Organ Chip microfluidic device. a Photograph (left), schematic illustration (center), and immunofluorescence micrographs (right) of a 2-channel microfluidic Organ Chip with iPS-BMVECs cultured on all surfaces of the basal vascular channel, and primary human brain astrocytes and pericytes on the upper surface of the central horizontal membrane in the apical parenchymal channel. At the top right, $z$-stack images of the pericytes (yellow, F-actin staining) and astrocytes (white, GFAP staining) in the top channel of the BBB Chip are reconstituted and shown from above; a side view of similar stacked images for the lower vascular channel containing BMVECs (blue, ZO-1 staining) is shown at the bottom right. A video of overlay z-stack reconstituting this 3D BBB can be seen in Supplementary Movie 1. b Timeline for the in vitro differentiation of the human iPS-BMVECs, and seeding in the BBB Chips. c Immunofluorescence micrographs of the human brain endothelium cultured on-chip for 3 days labeled with ZO-1, Claudin5, PECAM-1, GLUT-1, and P-glycoprotein (bar, $20 \mu \mathrm{m}$ ). d Electron micrographs of the human brain microvascular endothelium after 3 days in the BBB Chip, highlighting the presence of well-formed tight junctions (top, arrow) and adherens junctions (bottom, arrow) (bar, $100 \mathrm{~nm}$ ). e Low (left) and high (right) magnification immunofluorescence micrographic views of the human brain microvascular endothelium cultured on-chip for 3 days viewed from above demonstrating high levels of expression of ZO-1 and GLUT-1 across the entire endothelial cell monolayer (bar, $50 \mu \mathrm{m})$

Chip was cultured for one additional day under hypoxia to promote endothelial monolayer formation and to acclimate the cells to the device before being shifted to normoxic conditions for the remainder of the experiment and before initiating continuous medium flow $\left(100 \mu \mathrm{Lh}^{-1}\right)$ (Fig. 1b). We also controlled flow through the vascular channel to maintain physiological levels of fluid shear stress $\left(6\right.$ dyne $\mathrm{cm}^{-2}$ at $\left.100 \mu \mathrm{L} \mathrm{h}^{-1}\right)$ and a blood-like viscosity (3-4 cP; modified by adding $3.5 \%$ dextran to the medium).

Confocal immunofluorescence microscopic analysis after 3 days of microfluidic culture revealed that these conditions resulted in formation of an iPS-BMVEC monolayer that covered all four walls of the lower channel, creating a hollow vascular lumen, in addition to being interfaced directly across the porous ECMcoated membrane with primary human pericytes and astrocytes in the CNS channel above (Fig. 1a, Supplementary Movie 1). Higher magnification views revealed that the astrocytes extended processes through the $2 \mu \mathrm{m}$ pores of the PET membrane, and thus, came into direct contact with the luminal surface of the brain endothelium below (Supplementary Movie 2). These hypoxia-induced iPS-BMVECs also formed well developed tight junctions containing ZO-1 and Claudin-5, and expressed high levels of the cell-cell adhesion protein PECAM-1 along their lateral borders, as well as GLUT-1 and P-gp transporters on their 
apical cell membrane (Fig. 1c). Electron microscopic analysis confirmed that the differentiated iPS-BMVEC monolayer also displayed well-developed tight junctions and adherens junctions with characteristic morphology along the membrane-membrane interfaces between adjacent endothelial cells (Fig. 1d). Under these flow conditions, the endothelial monolayer within the human BBB Chip maintained its tight junctional integrity and continued to express high levels of ZO-1 as well as the glucose transporter GLUT-1 - the major nonneuronal glucose transporter in brain-on their apical surface for at least 1 week in culture, as detected by immunofluorescence microscopy (Fig. 1e).

Hypoxia-induced enhancement of brain-specific endothelial cell differentiation was also observed when gene and protein expression levels of representative BBB markers were compared with iPS-BMVECs cultured on-chip differentiated under hypoxic versus normoxic conditions. Statistically significant increases in mRNA expression were observed for genes encoding GLUT-1, insulin receptor protein (INSR), and the BBB efflux transport proteins P-gp, BCRP (breast cancer resistant protein), and multidrug resistance proteins 1 and 4 (MRP1 and MRP4), as well as endothelial cell-specific VE-cadherin (Supplementary Figure $2 \mathrm{a}$ and Supplementary Table 3). Increases in P-gp and BCRP expression were also confirmed by western blot analysis (Supplementary Figure $2 \mathrm{~b}$ and Supplementary Table 3). Moreover, mass spectrometric (MS) analysis of iPS-BMVECs collected from $\mathrm{BBB}$ Chips on the 3rd day of seeding revealed higher protein levels of ATP-binding cassette (ABC) and solute carrier (SLC) transporter proteins in hypoxia-differentiated brain endothelium cultured in the BBB Chips compared with endothelium differentiated under normoxic conditions (Fig. 2a, Supplementary Figure 3 and Supplementary Table 4). These results are similar to those from previous proteomic studies that also demonstrated enrichment of BCRP1, MRP1, and MRP4 proteins in the BBB relative to other microvessels in animal studies ${ }^{30}$. In addition, MS analysis revealed that the hypoxia-induced iPS-BMVECs deposit their own basement membrane ECM containing higher levels of collagen IV, laminin, and perlecan (basement membrane-specific heparan sulfate proteoglycan), fibronectin, SPARC, and agrin compared with when differentiated under normoxic conditions (Supplementary Table 5); these molecules are also found within the basement membrane of the human BBB in vivo ${ }^{31}$. Furthermore, to determine the contribution of astrocytes and pericytes to the function of iPS-derived human brain microvascular endothelium, we compared the mRNA profiles of iPS-BMVECs in mono-culture versus co-culture under continuous flow in the microfluidic BBB Chip. The mRNA levels of INSR, VE-cadherin, MRP1, and ZO-1 were all significantly higher in iPS-derived microvascular endothelium when co-cultured with brain astrocytes and pericytes on-chip (Supplementary Figure 4).

Reconstitution of in vivo levels of BBB function on-chip. Importantly, the higher expression of BMVEC-specific surface markers we observed in the presence of pericytes and astrocytes (Fig. 1, Supplementary Figure 4) was associated with enhanced barrier function, as quantified by measuring TEER on-chip using BBB Chips that contained integrated electrodes ${ }^{32}$. This method generates results as impedance in Ohms $(\Omega)$ rather than resistance $\left(\Omega \cdot \mathrm{cm}^{2}\right)$ because it is not normalized for surface area. These studies revealed that the impedance values of BBB Chips created with iPS-BMVECs differentiated under hypoxic conditions along with astrocytes and pericytes were $\sim 25,000 \Omega$ (Fig. 2b), which is 2 orders of magnitude higher than levels previously reported for BBB chips containing primary human BMVECs along with the brain perivascular cells $(\sim 400 \Omega)$ (Supplementary Figure 5). In contrast, while chips containing iPS-BMVECs established under normoxic conditions displayed a similar high maximum a

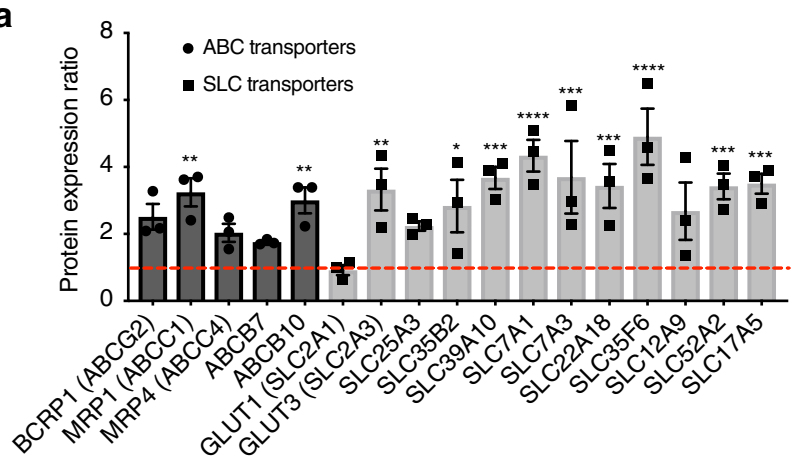

b

BBB-chip

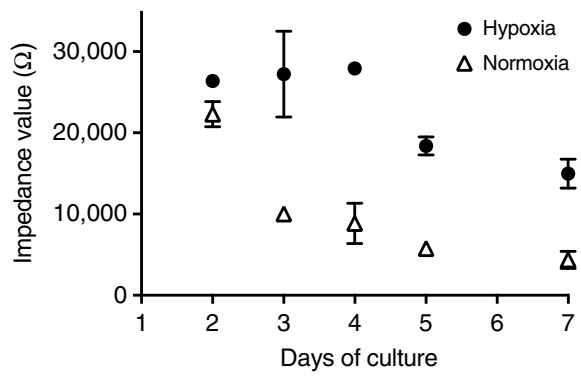

C

BBB-transwell

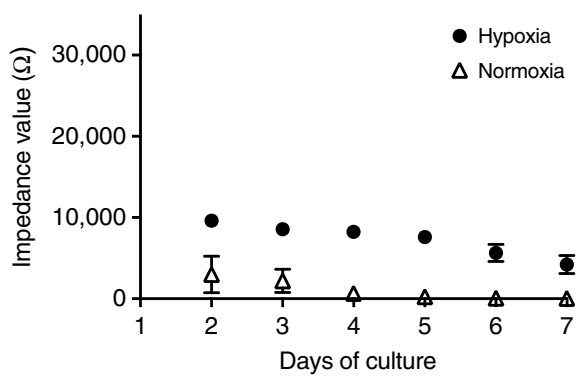

Fig. 2 Physical barrier functions of the human BBB reconstituted on-chip. a Graph showing protein expression ratios of $A B C$ and SLC transporter proteins in the iPS-hBMVECs differentiated under hypoxic conditions relative to normoxic conditions. $\mathbf{b}$ Impedance values for the human $B B B$ measured in the TEER chip over the first 7 days of culture on-chip (recorded in the frequency range of $0.1 \mathrm{~Hz}-100 \mathrm{kHz}$ ) with iPS-BMVECs that were differentiated under hypoxia (closed circles) or normoxia (open triangles). c Impedance values for the BBB formed by the same human astrocytes, pericytes, and iPS-hBMVECs that were differentiated under hypoxia (closed circles) or normoxia (open triangles) but cultured in static Transwell inserts measured by TEER measurement machine (EVOM2). Data are represented as $\pm \mathrm{SE}, N=3$. Statistical analysis is two-way ANOVA with Tukey's multiple comparisons test; ${ }^{\star} P<0.05$; ${ }^{\star \star} P<0.001 ;{ }^{\star \star \star} P<0.0001$

impedance value of $\sim 24,000 \Omega$ at 2 days after plating, barrier levels dropped by more than $50 \%$ by day 3 , and by $>80 \%$ at 1 week (Fig. 2b). These latter results were nearly identical to those previously reported by others who cultured iPS-BMVECs established under normoxic conditions in either Transwell inserts or Organ Chip devices ${ }^{13,33}$. We independently confirmed these results using fluorescently labeled dextran tracers to measure barrier integrity. The apparent permeability $\left(P_{\text {app }}\right)$ of the $\mathrm{BBB}$ Chip lined by hypoxia-induced iPS-BMVECs, astrocytes and pericytes was very low $\left(\sim 10^{-8}-10^{-9} \mathrm{~cm} \mathrm{~s}^{-1}\right)$, and the $P_{\text {app }}$ value inversely correlated with the size of the tracer (average $P_{\text {app }}=$ 
$\sim 8.9,1.1$, and $0.24 \times 10^{-8} \mathrm{~cm} \mathrm{~s}^{-1}$ for 3,10 , and $70 \mathrm{kDa}$ dextrans, respectively) (Supplementary Figure 6), as previously demonstrated in vitro and in vivo ${ }^{34,35}$. It is important to note that fluid flow also appeared to be required to sustain barrier function over time as we did not observe this level of barrier development when the same hypoxia-induced BMVECs were interfaced with astrocytes and pericytes cells in static Transwell cultures (Fig. 2c).

Furthermore, when we treated BBB Chips lined with iPSBMVECs differentiated under normoxic conditions with the HIFla inducer $\mathrm{CoCl}_{2}(100 \mu \mathrm{M})$, we produced nearly identical enhancement of TEER and similar results were obtained with another chemical hypoxia mimetic, dimethyloxalylglycine (DMOG; $100 \mu \mathrm{M}$ ) (Supplementary Figure 7). The highly enhanced, in vivo-like level of barrier function $(>20,000 \Omega)$ obtained with iPS-derived hBMVECs differentiated using $\mathrm{CoCl}_{2}$ also could be prolonged for more than 2 weeks when cultured under continuous flow in the microfluidic BBB Chip (Supplementary Figure 8a). In contrast, this high level of barrier function again was not obtained when the same cells were interfaced with astrocytes and pericytes under static conditions in Transwell cultures (Supplementary Figure 8b). Confocal immunofluorescence microscopic analysis of the $\mathrm{CoCl}_{2}$-activated human $\mathrm{BBB}$ Chips also confirmed that high levels of expression of ZO-1 and GLUT-1 were sustained on the surface of the iPS-BMVECs in these cultures (Supplementary Figure 9).

An in vitro model to study molecular trafficking across the BBB. Next, we tested if the enhanced human BBB Chip containing hypoxia-differentiated iPS-BMVECs, astrocytes and pericytes can recreate a functional metabolic barrier that regulates molecular traffic across the BBB, and if this occurs in a more physiologically relevant manner than in chips containing cells differentiated under normoxic conditions. The array of $\mathrm{ABC}$ transporters in the $\mathrm{BBB}$, which includes P-gp, MRP1 \& 4, and $\mathrm{BCRP}$, provides an important defense mechanism that actively secretes molecules leaked out of blood back into the capillary lumen in order to protect the brain ${ }^{36,37}$. As we found that all of these $A B C$ efflux pumps are expressed on the apical surface of the human iPS-BMVECs induced by hypoxia (Fig. 1c, Fig. 2a), we next analyzed their functionality and substrate selectivity. The hypoxia induction method was used for this and all subsequent studies because small molecule inducers may not mimic all of the effects of the hypoxic environment ${ }^{38,39}$, and thus, their use would require further system characterization.

To assess P-gp activity in the BBB Chip, we pretreated the chips with the P-gp inhibitor, verapamil, and then the known Pgp substrates, rhodamine 123 and $\mathrm{DiOC} 2^{40}$ as well as the drug citalopram, were perfused through the endothelium-lined vascular channel of the BBB Chips. Transcytosis of the P-gp substrate molecules with and without P-gp inhibitor treatment was measured under the same continuous flow conditions (100 $\mu \mathrm{L} \mathrm{h}^{-1}$ ), and hence shear stress, in both channels; thus, there was no difference in the unstirred water layer between the chips. Transcytosis of the P-gp substrate molecules was also carried out under static conditions using the BBB Transwell model on the second day after the cell seeding when the barrier integrities were similar to the BBB Chip model $\left(3 \mathrm{kDa}\right.$ Dextran $P_{\text {app }}$ values $=$ $\sim 2 \times 10^{-7} \mathrm{~cm} \mathrm{~s}^{-1}$ ). The $P_{\text {app }}$ of rhodamine 123 and DiOC2 were calculated by measuring changes in their fluorescent intensities between the two channels; MS analysis was used to measure the permeability of citalopram.

These studied revealed significant enhancement of the permeability of all three of these molecules through the BBB on-chip when the P-gp efflux pump was blocked by its inhibitor, verapamil, whereas a significant increase in the permeability of these molecules could not be detected when the same cells were interfaced to form a BBB in static Transwell cultures (Fig. 3b). Remarkably, citalopram was also shown to increase its permeability when P-gp was inhibited in the microfluidic BBB Chip (Fig. 3a), which recapitulates similar in vivo findings shown in past animal studies ${ }^{10,41}$. This is an important result because all past in vitro BBB models failed to identify citalopram as a P-gp substrate 42,43 .

To analyze the activities of BCRP and MRP1 transporter proteins, the BBB Chips were pretreated with inhibitors of various ABC transporter inhibitors, including verapamil MK571, and Ko143 that target P-gp, the MRPs and BCRP, respectively, and then uptake of fluorescent substrates, rhodamine 123 and DiOC2, was quantified under flow using fluorescent dextran $(3 \mathrm{kDa})$ to monitor barrier integrity. When we measured the $P_{\text {app }}$ of these two $\mathrm{ABC}$ transporter substrates in BBB Chips containing iPSBMVECs differentiated under hypoxic conditions along with astrocytes and pericytes, we found that inhibition of P-gp with verapamil resulted in significant influx of rhodamine 123 and DiOC2 into the CNS channel, and inhibition of BCRP with Ko143 had a small but significant effect on transport of DiOC2, but no effect on rhodamine 123 , as previously described in other models $^{13,44}$ (Fig. 3c). In contrast, when similar studies were carried out with BBB Chips generated under normoxic conditions, we observed an increase of rhodamine 123 in the CNS channel when MRP1 was inhibited, but no change when P-gp was inhibited, and DiOC2 influx was not affected by any of the inhibitors (Fig. 3c). As P-gp is the main efflux pump for rhodamine $123^{45,46}$ and DiOC2 influx was modulated in a manner similar to what is observed in vivo ${ }^{45}$ in the hypoxic chips, these studies demonstrate that while the iPS-BMVECs differentiated under normoxic conditions may generate an effective permeability barrier ${ }^{13,47}$, they do not fully recapitulate the human BBB's specialized molecular transport functions that are highly relevant to the drug development process.

To further investigate the potential of the hypoxia-induced human BBB Chip as an in vitro tool to study brain transporterdependent drug efflux, we tested the cancer drug doxorubicin (Dox). While Dox is used to treat many types of cancer, its efficacy for brain tumors is limited due to poor penetration of the drug through the BBB because it is pumped back out of the endothelium and into the blood predominantly by P-gp transporters ${ }^{48}$, and less by MRP $1^{49}$ or BCRP 50 . Consistent with these in vivo observations in humans, we found that inhibition of P-gp with Verapamil induced more than a 2.7 -fold increase in Dox influx into the CNS channel (rise in $P_{\text {app }}$ from 1.4 to $3.7 \times$ $\left.10^{-8} \mathrm{~cm} \mathrm{~s}^{-1}\right)$ when added under flow $\left(100 \mu \mathrm{L} \mathrm{h}^{-1}\right)$ through the vascular channel of the BBB Chip (Fig. 3c), which is similar to the $\mathrm{P}$-gp efflux ratio observed in vivo ${ }^{37}$. Again, as observed in vivo, inhibition of MRP1 or BCRP did not alter the permeation of Dox into the CNS channel (Fig. 3c). Together, these results suggest that differentiation of iPS-BMVECs in the presence of hypoxia, and positioning of these cells under physiological flow at a tissuetissue interface with human brain pericytes and astrocytes, generates of an artificial human $\mathrm{BBB}$ that exhibits higher substrate specificity and functionality of the efflux barrier than previously reported in any in vitro BBB model.

Reversible osmotic opening of the human BBB on-chip. Hyperosmolar agents, such as high concentration mannitol solutions, are used clinically to overcome the BBB permeability limitation in order to increase delivery of drugs to the brain 51,52 Intravenous infusion of a mannitol solution induces reversible opening of pores within the BBB with a radius of about $200 \AA$ in animals $^{53}$ and humans ${ }^{53,54}$, and this recovers due to bulk fluid flow from the blood to the brain ${ }^{54}$. We therefore explored whether we can use our hypoxia-enhanced BBB Chip to model 
a

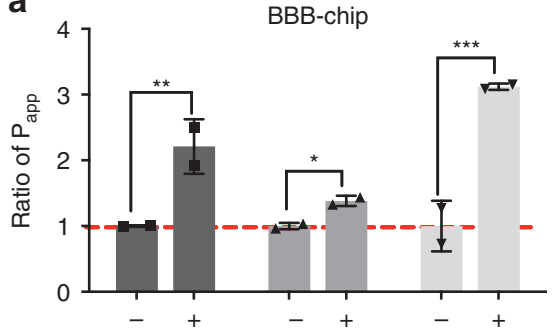

Addition of P-gp inhibitor b

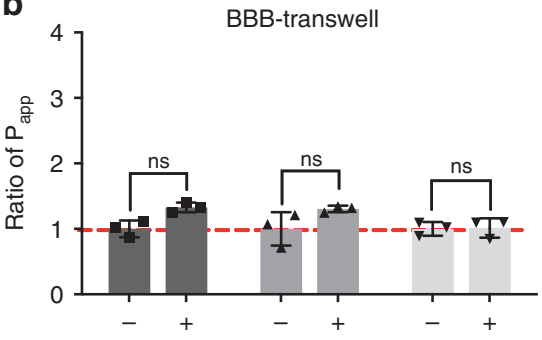

Addition of P-gp inhibitor
- Rhodamine

$\triangle$ DiOC2

- Citalopram
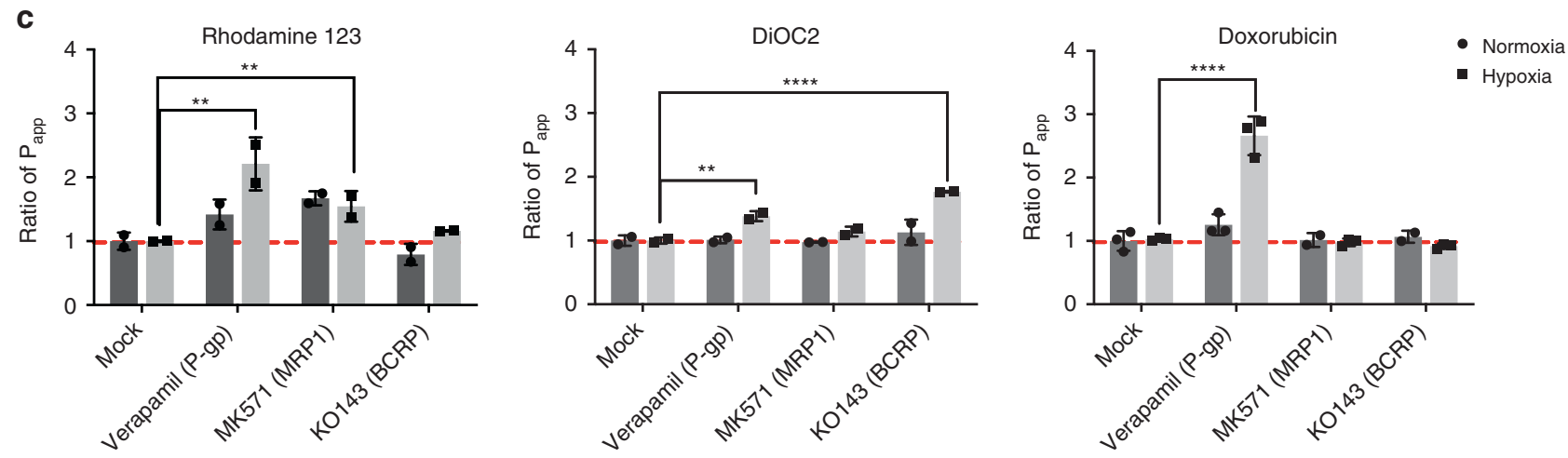

Fig. 3 Metabolic barrier function of the human BBB Chip. P-gp activities measured in the BBB Chip (a) versus BBB Transwells (b) that were pretreated with verapamil to inhibit the P-gp activity in the presence or absence of the P-gp substrates, rhodamine 123, DiOC2, or citalopram. c BBB efflux pump substrate selectivity and functionality measured in the BBB Chips using rhodamine 123 as a substrate of P-gp and MRP1 (left); DiOC2 as a substrate of P-gp and BCRP (middle); and doxorubicin interacting with P-gp (right) with or without addition of efflux transporter inhibitors (verapamil for P-gp, MK 571 for MRPs, and Ko143 for BCRP). Ratio of $P_{\text {app }}$ indicates fold changes in the apparent permeability $\left(P_{\text {app }}\right)$ of molecules resulting from chemical modulation of specific efflux pump activity. Data are presented as means $\pm S E, N=2$ for the chip experiments for rhodamine $123, \mathrm{DiOC2}$, and citalopram, as well as $N=3$ for the BBB Transwell experiments and BBB chip experiments for Doxorubicin. The statistical analysis is two-way ANOVA with Tukey's multiple comparisons test; ${ }^{\star} P<0.05 ;{ }^{\star \star} P<0.001 ;{ }^{\star \star \star} P<0.0001$

delivery of large antibodies, such as those used as cancer therapeutics, by opening the barrier with hypertonic mannitol onchip. Hypertonic ( $485 \mathrm{mOsmol} \mathrm{L}^{-1}$ ) mannitol solution was flowed through the endothelium-lined vascular channel of the BBB Chip for $1 \mathrm{~h}$ to open the BBB, and then additional hypertonic medium containing $10 \mathrm{kDa}$ dextran and the anti-cancer therapeutic antibody, cetuximab, was flowed for an additional hour through the same channel (Fig. 4a).

Constant exposure to the hypertonic medium resulted in a 50\% drop in TEER within $2 \mathrm{~h}$, which gradually recovered to normal levels within $4 \mathrm{~h}$ following infusion of isotonic medium into the vascular channel (Fig. 4b). Osmotic opening of the BBB in this manner also resulted in increased penetration of both $10 \mathrm{kDa}$ dextran (Fig. 4c) and the cetuximab antibody (Fig. 4d) into the CNS channel, as measured by a rise in $P_{\text {app }}$ from 3 to $32 \times 10^{-8}$ $\mathrm{cm} \mathrm{s}^{-1}$ and from 2 to $5.1 \times 10^{-8} \mathrm{~cm} \mathrm{~s}^{-1}$, respectively. The hypertonic medium was replaced by isotonic medium containing the same compounds after $1 \mathrm{~h}$ to allow the BBB Chip to recover barrier function, and after an additional $30 \mathrm{~min}$, we found that the barrier integrity measured by TEER had not fully recovered (Fig. 4b), indicating that both the dextran and the antibody continued to penetrate through the $\mathrm{BBB}$ and into the CNS channel. However, the permeabilities of dextran (Fig. 4c) and the antibody (Fig. 4d) decreased to the normal range within $4 \mathrm{~h}$, resulting in full recovery of barrier integrity (Fig. 4b). To our knowledge, this is the first demonstration of delivery of a clinically approved antibody drug by reversible osmotic opening of the human BBB in vitro, which mimics responses seen in human patients ${ }^{52,54}$.
Recapitulation of BBB-shuttling activities on-chip. The BBB prevents the transport of nearly $100 \%$ of large drugs $(>500 \mathrm{Da})$ and antibodies $(150 \mathrm{kDa})$ into the brain, and thus, many laboratories are seeking ways to discover new brain-targeting molecules that could enable delivery of drugs across the BBB and into the CNS. A few brain shuttling molecules have been identified, including IgG antibodies ${ }^{55}$ and ligands for cell surface receptors on the brain microvascular endothelium that help drugs transit through the endothelium and into the CNS via receptormediated transcytosis ${ }^{56}$. Accurate in vitro assessment of CNStargeted drug delivery has not been possible previously because it requires that human BBB culture models provide a physiological barrier that prevents paracellular leakage, as well as expression of brain endothelial cell-specific receptors and transporters. Given the high level of differentiation of iPS-BMVECs in our hypoxia-induced human BBB Chip, we explored whether this improved model can be used to directly assess the human BBBpenetrating capacity of peptides, nanoparticles, and antibodies in vitro.

Angiopep-2 is a small peptide ligand of LRP-1, expressed by iPS-BMVECs (Supplementary Figure 10), that has been shown to penetrate the $\mathrm{BBB}$ in vitro and in vivo ${ }^{57}$. The brain penetrating capacity of multiple drugs, including paclitaxe ${ }^{58}$ and a cancer therapeutic HER-2 antibody ${ }^{59}$, has been shown to be enhanced when modified with Angiopep-2. When we flowed Q-dot nanoparticles $(20 \mathrm{~nm})$ coated with Angiopep-2 through the endothelium-lined vascular channel of the human BBB Chip, these particles exhibited. Approximately 3.5 -fold greater capacity to penetrate into the CNS channel compared with Q-dots 
a

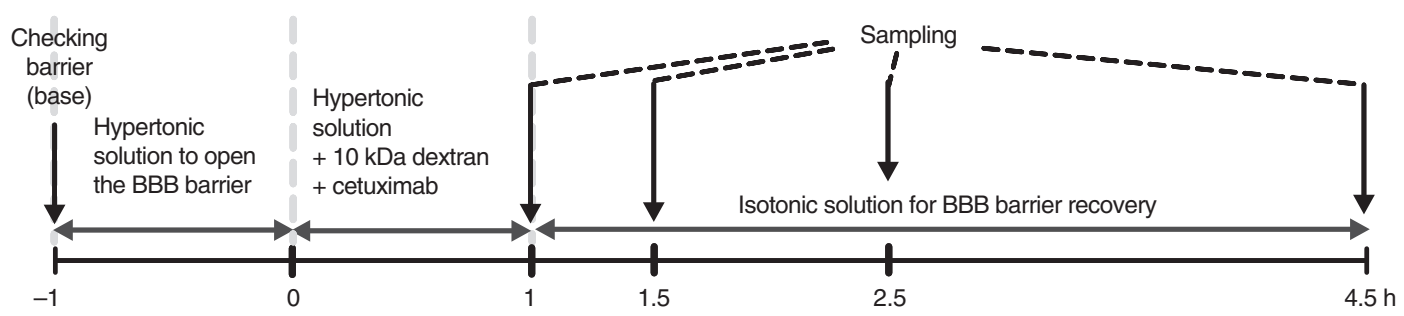

b

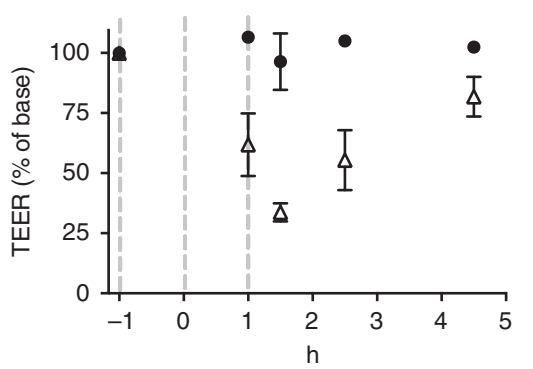

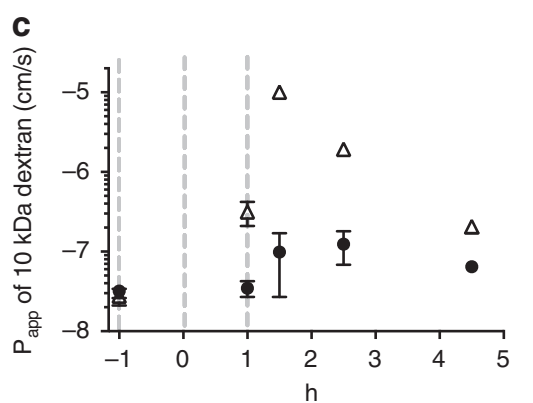

d

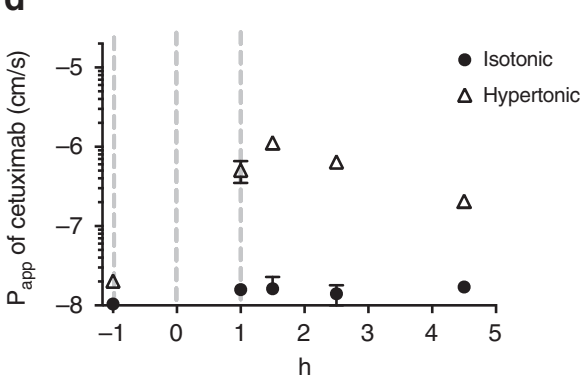

Fig. 4 Penetration of a therapeutic antibody through the BBB Chip using osmotic solutions. a Experimental design for assessing osmotic opening of endothelial barrier in the BBB chip, including the timeline for medium (hypertonic and isotonic) changes, and drug and tracer dosing, as well as sampling times. Barrier integrity of the BBB Chips under isotonic and hypertonic conditions as monitored by measuring TEER (b) or $P_{\text {app }}$ of $10 \mathrm{kDa}$ dextranconjugated to tetramethylrhodamine (c) or of the therapeutic antibody cetuximab (d). Data are presented as means $\pm \mathrm{SE}, N=2$ representing independent BBB Chips

a

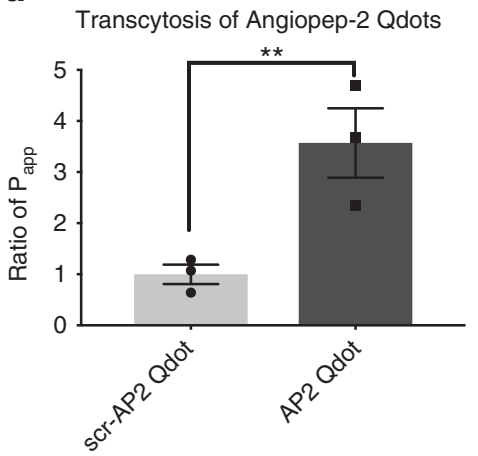

b

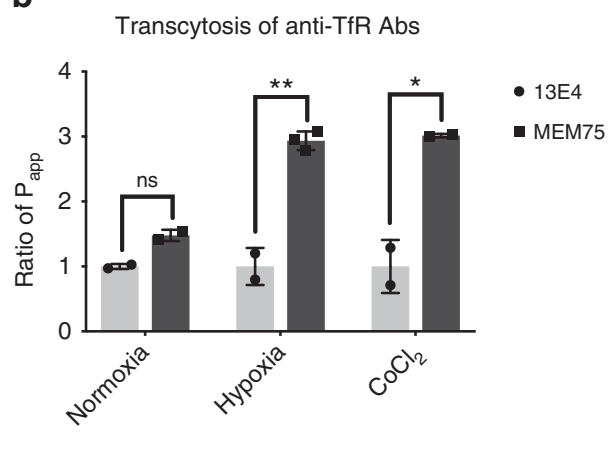

Fig. 5 Recapitulation of Angiopep-2 peptide and anti-TfR antibody shuttling through the BBB on-chip. a Transcytosis of the known BBB shuttle molecule, Angiopep-2, measured by quantifying the Ratio of $P_{\text {app }}$ of Angiopep-2-conjugated quantum dot (Q-dot) nanoparticles versus Q-dots coated with a scrambled version of the Angiopep-2 peptide in chips lined by iPS-BMVECs differentiated under hypoxic conditions. $N=3$, representing independent BBB Chips. b Ratio of $P_{\text {app }}$ of the known BBB-shuttling low-affinity anti-TfR antibody MEM75 (dark gray) relative to that displayed by the non-shuttling highaffinity anti-TfR 13E4 antibody (light gray) measured in human BBB Chips generated with iPS-BMVECs differentiated under normoxic or hypoxic conditions, or in the presence of $\mathrm{CoCl}_{2}$. Data are presented as means $\pm \mathrm{SE}, \mathrm{N}=2$ representing independent BBB Chips. The statistical analysis is two-way ANOVA with Tukey's multiple comparisons test; ${ }^{\star} P<0.05$; ${ }^{\star \star} P<0.001 ;{ }^{\star \star \star} P<0.0001$

coated with a scrambled peptide (Fig. 5a), even though there was no significant change in barrier integrity (Supplementary Figure 11a).

The transferrin receptor (TfR) on BMVECs (Supplementary Figure 10) also has been shown to function as a molecular shuttle $^{60}$ that can mediate differential penetration of anti-TfR antibodies through the BBB that differ in their binding affinity. More specifically, the anti-TfR antibody MEM75 has been shown to penetrate into the brain more efficiently than the $13 \mathrm{E} 4$ antibody in vitro ${ }^{61}$. This is because MEM75 has a reduced affinity for the receptor at $\mathrm{pH} 5.5$, while the $13 \mathrm{E} 4$ anti-TfR antibody exhibits a $\mathrm{pH}$-independent affinity that results in its entrapment within intracellular vesicles in brain endothelial cells. In past studies with in vitro human BBB models, including studies with human iPS-BMVECs in Transwell cultures, it was not possible to replicate this highly clinically relevant form of shuttling across the $\mathrm{BBB}$ because of the poor barrier function and high levels of paracellular influx ${ }^{61,62}$, and we obtained similar results when MEM75 or 13E4 antibodies were flowed through the vascular channel with the BBB Chip containing endothelium differentiated under normoxic conditions (Fig. 5b). We also did not observe any significant difference in transcytosis ability between these two TfR antibodies when we flowed the antibodies through BBB Chips lined by primary (as opposed to iPSC-derived) human BMVECs (Supplementary Figure 12), which produced only a minimal ( 1.4-fold) difference in transcytosis abilities for the two TfR antibodies much as previously observed in past microfluidic primary $\mathrm{BBB}$ models ${ }^{63}$. In contrast, when these anti-TfR 
antibodies were flowed through the vascular channel of the hypoxia-enhanced BBB Chip, we were able to demonstrate threefold higher penetrance of MEM75 into the CNS channel relative to the $13 \mathrm{E} 4$ antibodies that was previously predicted in vitro $^{61}$ (Fig. 5b), and this was accomplished without compromising general barrier integrity (Supplementary Figure 11b). In addition, BBB Chips formed with iPS-BMVECs induced by exposure to $\mathrm{CoCl}_{2}$, exhibited similar differential transcellular transport, again capturing the threefold difference between the two anti-TFR antibodies (Fig. 5b) without disrupting barrier integrity (Supplementary Figure 11c).

\section{Discussion}

Taken together, these results demonstrate that mimicking the hypoxic microenvironment of the developing brain during differentiation of human iPSCs into BMVECs, by either lowering oxygen levels or adding HIFla-inducing mimetics (e.g., $\mathrm{CoCl}_{2}$ or DMOG), enables differentiation of endothelial cells that recapitulate human-relevant physiological BBB properties when the cells are interfaced with human brain astrocytes and pericytes in a 2-channel microfluidic BBB Chip. This is the first human BBB Chip model that permits analysis of the BBB-penetrating activities of known BBB shuttle peptides, as well as analysis of the TfRbased antibody shuttling mechanism ${ }^{61}$, under physiologically relevant flow conditions in vitro. In addition, this enhanced BBB Chip replicates the delivery of a clinically approved therapeutic antibody (cetuximab) by reversible osmotic opening of the human BBB in vitro, as is observed in human patients. Thus, while past work in this field described other human BBB models that contained similar cells maintained under different culture conditions (e.g., single channel microfluidic devices ${ }^{64}$, 3D tubelike cultures ${ }^{6,65}$ ), none of them demonstrated these various clinically relevant functionalities (e.g., in vivo-like barrier function for 2 weeks compared with 2-3 days in past studies) and technical capabilities (e.g., real-time measurements of barrier function using integrated TEER electrodes). These functional properties are crucial for a preclinical drug development tool that can be used for discovery of new BBB shuttles and brain-targeted therapeutics, which was the specific focus of this study.

The human BBB Chip containing human iPS-BMVEC differentiated under developmentally inspired hypoxic conditions exhibited greatly increased and sustained barrier function than past in vitro $\mathrm{BBB}$ models with TEER levels similar to those estimated for the human $\mathrm{BBB}$ in vivo ${ }^{66}$. Moreover, this enhanced induction of brain-specific endothelial cell differentiation by hypoxia is mediated by HIF1 $\alpha$, and the hypoxia conditioning step can be circumvented by artificially increasing HIF1a levels using small molecules $\left(\mathrm{CoCl}_{2}\right.$ or DMOG) that have been previously shown to mimic the effects of hypoxia under normoxic conditions ${ }^{39,67}$.

We and others have previously developed 3D human BBB Chip models using immortalized, primary, or iPS-derived BMVECs, but they either fail to generate in vivo-like barrier function or rapidly lose their phenotypic properties $6,13,68,69$. Another microfluidic BBB Chip was developed as a potential tool for drug testing that contains human iPS-BMVECs derived under normoxic conditions and cultured under continuous flow ${ }^{35}$; however, those cells were interfaced with rat (rather than human) astrocytes, there were no pericytes in that model, and active barrier function specifically mediated by active efflux pumps was not fully assessed. Another microfluidic BBB model was developed utilizing iPS-BMVECs that provides a tight barrier function for up to 6 days $^{64}$, but this model only contains a single microfluidic channel, which makes it difficult to carry out TEER measurements, explore the contributions of parenchymal cells, or test for the transcytotic delivery of different drugs from capillary channels to brain side. A self-organized microvascular model of the BBB utilizing human iPS-BMVECs and human primary astrocytes and pericytes cultured within a fibrin gel has been developed $^{65}$; however, utilization of fibrin gel makes it difficult to incorporate electrodes for TEER measurements to quantitatively and continuously assess barrier function with high sensitivity, as done in our 2-channel human BBB Chip. Diffusion of small molecules into the fibrin gel, and long residence time, also makes it difficult to accurately and quantitatively measure transBBB permeability values when using fluorescent tracers. The advantage of the hypoxia-enhanced $\mathrm{BBB}$ Chip in which the differentiated human brain microvascular endothelium also experiences dynamic fluid flow and shear stress that significantly influence BBB structure and function ${ }^{70}$ is that it sustains in vivolike barrier functions, as well as tightly regulated transcellular transport activities that are crucial for drug development studies, for much longer period of time (at least 2 weeks) compared with past human $\mathrm{BBB}$ models. The 2-channel design of the human BBB Chips also enables direct and independent access to both the parenchymal and vascular compartments, as well as real-time TEER measurements for quantitative assessment of barrier function, which greatly facilitates trans-BBB shuttling studies. Hence, the human BBB Chip should provide a more accurate model for assessing the ability of chemicals, CNS therapeutics, and drug targeting vehicles to pass through the human BBB and enter the brain.

The enhanced differentiation conditions we applied were inspired by the observation that the BBB forms in a hypoxic environment during embryological development ${ }^{20,22,38}$, and this is accompanied by expression of Wnt7a/Wnt7 $\mathrm{b}^{25,26}$, as well as HIF $1 a^{27,28}$. Interestingly, when we differentiated the human iPSCs under similar hypoxic conditions, they expressed a similar developmental program including expression of Wnt7a and HIF1a, indicating that hypoxia triggers these changes in vitro as well. Importantly, this response was also accompanied by upregulation of various biomarkers associated with brain neovascularization including VEGF, GLUT-1, VE-cadherin, and PECAM during the differentiation of iPS-BMVECs, as well as improved formation of cell-cell junctions and membrane transporters that resulted in significantly improved $\mathrm{BBB}$ function that was stable over longer times than observed in past models. But induction of HIF1 $\alpha$ appears to be the essential trigger for the generation of an enhanced in vivo-like BBB Chip as similar induction of iPSBMVEC differentiation could be obtained by addition of small molecule inducers of HIFla $\left(\mathrm{CoCl}_{2}\right.$ and DMOG). In fact, treatment with these hypoxia mimetics may prove to be a useful method to generate improved iPSC-derived BBB Chips without requiring a specialized hypoxic chamber. It is also interesting to note that this hypoxia-induced differentiation response appears to be specific for the human iPSCs as exposure to human primary adult BMVECs to hypoxia results in the opposite response (e.g., increased levels of HIF1a induce expression of VEGF, which leads to higher BBB permeability and edema $)^{71,72}$. In contrast, in the hypoxia-induced iPS-BMVECs, this response was not observed likely because HIF1a levels decreased drastically when the differentiated hBMVECs were seeded in the chip.

Using the hypoxia-enhanced human BBB Chip, we were able to demonstrate that many clinically relevant responses of the $\mathrm{BBB}$ can be recapitulated in vitro. The first and most important is maintenance of sustained levels of low barrier permeability similar to those observed in human brain for more than 2 weeks in vitro. We also showed that the $\mathrm{BBB}$ Chip can be used to study reversible opening of the $\mathrm{BBB}$ using hypertonic solutions in vitro, as is done in vivo; this technique may be used to develop improved therapeutic delivery strategies in the future, as 
demonstrated here by delivery of an FDA approved therapeutic antibody, mimicking a clinically relevant drug delivery strategy 52 . Even more importantly, the enhanced BBB chip was able to mimic transporter-mediated drug efflux including appropriate substrate specificity. Using our enhanced BBB Chip, we first recapitulated in vivo interaction of $\mathrm{P}$-gp and citalopram ${ }^{10,41}$ under physiological flow, which could not be reproduced under static conditions in past 3D models. Therefore, our BBB Chip model demonstrated the advantage of using microfluidic system to mimic in vivo cellular transport of molecules. Furthermore, in vivo shuttling of CNS-targeting peptides, nanoparticles, and antibodies across the BBB were recapitulated using enhanced BBB Chip, enabling examination of CNS-targeting drug delivery systems as well.

In summary, the human BBB Chip created using iPS-BMVECs exposed to developmentally inspired hypoxic differentiation conditions and cultured under physiological flow when interfaced with human brain pericytes and astrocytes exhibits enhanced functionalities relative to past human BBB models. These functionalities provide long-term improvements resulting in formation of a stable BBB with high, in vivo-like permeability restriction that lasts up to 2 weeks; high levels of expression of tight junction, SLC and ABC proteins; and proper function of efflux proteins, as well as drug, peptide, nanoparticle, and antibody transcytosis capabilities that are dependent on TfR and LRP1 surface proteins. This enhanced human BBB Chip provides a significant advance as it allows for improved drug screening by better recapitulating the in vivo environment of the BBB. It may therefore prove useful for development of drugs or delivery vehicles that selectively cross the BBB and target the CNS, as well as for modeling CNS diseases in vitro using patient-derived iPSCs to investigate improved brain therapies and advance personalized medicine.

\section{Methods}

Cell culture. The human iPS cell line IMR90-4 (WiCell Research Institute) was propagated on tissue-culture plate that were coated with Matrigel (BD Biosciences) by using mTeSR1 medium (Stem Cell Technology) and maintained according to WiCell Feeder Independent Pluripotent Stem Cell Protocols provided by the WiCell Research Institute (http://www.wicell.org). Primary human astrocytes isolated from cerebral cortex were obtained from ScienCell and maintained in Astrocyte medium (ScienCell). Primary human brain pericytes were also obtained from ScienCell and maintained in Pericyte medium (ScienCell). The primary cells were used at passage 3-6.

BMVEC differentiation. Human iPS cells were differentiated to hBMVEC as previously described ${ }^{33}$ with modification of $\mathrm{O}_{2}$ conditions. Briefly, IMR90-4 iPSCs (WiCell Research Institute, Inc.) were dissociated using Accutase and seeded on 6well plate coated with Matrigel at a concentration of $1.8 \times 10^{5}$ cells per well. Cells were cultured with mTeSR 1 media for three days until the concentration reaches to $2.5 \times 10^{5}$ cells per well. The human iPSCs were differentiated to a mixed endothelial cells and neural progenitor cell culture by switching cells to unconditioned medium (UM) for 6 days (D0-D6). UM includes $392.5 \mathrm{~mL}$ DMEM/F12 (Invitrogen), 100 $\mathrm{mL}$ of Knockout Serum Replacement (KOSR) (Thermo Fisher Scientific), $5 \mathrm{~mL}$ of non-essential amino acids (Invitrogen), $2.5 \mathrm{~mL}$ of Glutamax (Invitrogen), and 3.5 $\mu \mathrm{L}$ of $\beta$-mercaptoethanol (Sigma). The endothelial cells were selectively expanded by switching to endothelial cell (EC) media supplemented with retinoic acid (RA) (D7-D8). For steady $5 \% \mathrm{O}_{2}$ conditions during the differentiation (from D0 to D9), cells were cultured in the incubator (Heracell ${ }^{\mathrm{TN}} 150 \mathrm{i}$ Tri-Gas Incubator, Thermo Scientific) flushing with a $5 \% \mathrm{O}_{2}-5 \% \mathrm{CO}_{2}-\mathrm{N}_{2}$ balance continuously. To analyze the mRNA expressions during the BMVEC differentiation, total RNA was extracted from cell culture using RNeasy Mini Kit (Promega) and cDNA was synthesized using Maxima First Strand cDNA synthesis Kit according to the instruction's protocol. The multiplex qPCR was performed by using TaqMan probes (Thermo Fisher Scientific) in a QuantStudio 7 Flex (Thermo Fisher Scientific). Label-free proteomics were carried as described previously using mass spectrometry analysis $^{73}$.

Device fabrication. The design of the human BBB-on-a-chip was modified from the previously reported human Small Airway Chip ${ }^{74}$. Parts for apical and basal channels were cast in polydimethylsiloxane (PDMS) (Sylgard 184, Ellsworth Adhesives) at a 10:1 ratio of base to curing agent in custom 3D printed Prototherm molds (Proto Labs). The hollow microchannels were 2-cm long and 1-mm wide; top and bottom channels were 1 and $0.2 \mathrm{~mm}$ high, respectively. PDMS was degassed at $-80 \mathrm{kPa}$ until all bubbles were removed, and cured at $60{ }^{\circ} \mathrm{C}$ for at least $4 \mathrm{~h}$. Twenty-micrometer thick, transparent, track etched, polyethylene terephthalate (PET) membranes with $0.4-\mu \mathrm{m}$ perpendicular pores at a pore density of $4 \times 10^{6}$ pores $\mathrm{cm}^{-2}$, were purchased from A.R. Brown and laser cut to size and to add ports. Membranes were bonded to PDMS using an epoxy silane method modified from Tang and Lee ${ }^{75}$. Briefly, PDMS parts and membranes were treated with $\mathrm{O}_{2}$ plasma at $20 \mathrm{~W}$ for $45 \mathrm{~s}, \mathrm{O}_{2}$ gas flow $50 \mathrm{sccm}$ to $0.80 \mathrm{mbar}$. PDMS parts were submerged in a $1 \%$ (3-Glycidyloxypropyl)trimethoxysilane solution (Sigma) and membranes were submerged in a 5\% (3-Aminopropyl)triethoxysilane solution (Sigma) for $20 \mathrm{~min}$ at room temperature to bond silanes to the surface of the parts. Parts were rinsed in MilliQ filtered water and dried with compressed air. Parts were aligned and bonded by hand and placed in a $60^{\circ} \mathrm{C}$ oven under $500 \mathrm{~g}$ weights for at least $12 \mathrm{~h}$ to anneal the bond. Chips were inspected for delamination, debris, or other defects before use.

BBB reconstitution on a chip. PDMS surface of the chips were activated with oxygen plasma treatment and the channels were coated with collagen IV $\left(400 \mu \mathrm{g} \mathrm{ml}^{-1}\right)$ and fibronectin $\left(100 \mu \mathrm{g} \mathrm{ml}^{-1}\right)$ overnight. Both channels of the chip were rinsed with PBS and then with astrocyte media before seeding cells. For coculturing astrocytes and pericytes in the brain channel of the BBB Chip, a density of $0.7 \times 10^{6} \mathrm{cells} \mathrm{ml}^{-1}$ of human astrocyte and $0.3 \times 10^{6}$ cells $\mathrm{ml}^{-1}$ of pericyte were mixed together in the astrocyte media and seeded on the apical channel of the chip, then incubated in the incubator for $1 \mathrm{~h}$. To remove the access of the astrocyte and pericytes, channels of the chip were washed with EC + RA medium (endothelial medium with addition of fibroblast growth factor (Fgf) and retinoic acid (RA) ${ }^{33}$ ) and then $2.3 \times 10^{7}$ cells ml $^{-1}$ of iPS-BMVEC (D8) was seeded in the basal channel, and the device was flipped immediately to allow the BMVECs to adhere to the ECM-coated PET membrane. After $5 \mathrm{~h}$ incubation in the hypoxic incubator, the device was flipped back to let the rest of BMVECs sit on the bottom and sides of the channel to form a capillary lumen. Chips seeded with cells were maintained in the hypoxic incubator for $24 \mathrm{~h}$, and then the BBB Chip was fed with EC medium deprived of Fgf and RA and transferred to the regular incubator $\left(5 \% \mathrm{O}_{2}-5 \% \mathrm{CO}_{2}\right)$. On the second day of cell seeding on the microfluidic device, BBB Chips were attached to peristaltic pumps (microprocessor controlled dispensing pump from Ismatec), and EC medium was flowed through the channels $\left(60-100 \mu \mathrm{L} \mathrm{h}^{-1}\right)$ to allow the BBB Chips adjust to flow conditions; then on the third day of seeding dosing studies were performed. For TEER measurements, we seeded astrocyte, pericyte, and iPS-BMVEC on TEER chips ${ }^{32}$, and measured the four-point impedance using a PGstat $128 \mathrm{~N}$ from Metrohm Autolab BV as previously reported ${ }^{32}$ Apparent permeability $\left(P_{\text {app }}\right)$ of the barrier was calculated by following a previously described method ${ }^{73}$. Briefly, $100 \mu \mathrm{g} \mathrm{mL}^{-1}$ of dextran tracers were dosed through the vascular channels for a known period of time, and concentration of the dextran tracers in the outlet samples from both vascular and brain channels was determined by using BioTek (BioTek Instruments, Inc., Winooski, VT, USA). Then, following equation was used to calculate $P_{\text {app }}$ :

$$
P_{\text {app }}=\frac{V_{\mathrm{r}} \times C_{\mathrm{r}}}{A \times t \times \frac{\left(C_{\mathrm{d}-\text { out }} \times V_{\mathrm{d}}+C_{\mathrm{r}} \times V_{\mathrm{r}}\right)}{\left(V_{\mathrm{d}}+V_{\mathrm{r}}\right)}}
$$

Here, $V_{\mathrm{r}}$ is volume of receiving channel at time $t, V_{\mathrm{d}}$ is volume of dosing channel at time $t, A$ is area of membrane which is $0.167 \mathrm{~cm}^{2}$ in our chip model, $C_{\mathrm{r}}$ is measured concentration of tracer in the receiving channel, and $C_{\mathrm{d} \text {-out }}$ is measured concentration of tracer in the dosing channel effluent. Furthermore, we quantified the percent loss of the molecules into the chip materials with and without cells being present. We tracked the inlet dosing concentrations for both vascular and brain channels as well as outlet concentrations of both channels. Tracer loss (\%) was monitored and accounted for during the experiment using the following formula:

$$
\text { Tracer loss }(\%)=100 \times\left(1-\frac{\left(C_{\mathrm{d}-\text { out }} \times V_{\mathrm{d}}\right)+\left(C_{\mathrm{r}} \times V_{\mathrm{r}}\right)}{C_{\mathrm{d}-\text { in }} \times V_{\mathrm{d}}}\right)
$$

Here, $C_{\mathrm{d} \text {-out }}$ is the measured concentration of tracer in dosing channel effluent, $C_{\mathrm{d} \text {-in }}$ is the concentration of dosing medium in inlet, $C_{\mathrm{r}}$ is the measured concentration of tracer in receiving channel effluent. $V_{\mathrm{d}}$ is the volume of dosing channel effluent at time $t$, and volume of receiving channel effluent at time $t$

Immunofluorescence microscopy. BBB Chips were fixed with $4 \%$ paraf ormaldehyde in PBS for $10 \mathrm{~min}$ and then washed with PBS. Immunostaining was performed after permeabilization in PBS with $0.1 \%$ Triton X-100 (Sigma) and blocking for $30 \mathrm{~min}$ in $10 \%$ goat serum in PBS with $0.1 \%$ Triton X-100. Antibodies used in the study were listed in the Online Reporting Summary, which were 1:100 dilutions in $10 \%$ goat serum and incubated overnight on the BBB Chip at $4{ }^{\circ} \mathrm{C}$. Fluorescently conjugated secondary antibodies with Alexa Fluor-488, Alexa Fluor555, or Alexa Fluor-647 were then used when the primary antibodies are not conjugated. Conventional confocal imaging was carried out with a 405-laser diode, an Argon laser and a tunable white laser using a Leica SP5 X MP Inverted Laser Scanning Confocal Microscope with an $\sim 25 \AA$ water immersion objective and a Zeiss Axio Observer microscope. For electron microscopy images, BBB chips samples were embedded in LR white resin by following the protocol used in Harvard Medical School Electron Microscopy Facility. Briefly, the BBB Chip is 
washed in $0.1 \mathrm{M}$ cacodylate buffer and post-fixed with $1 \%$ osmium tetroxide $\left(\mathrm{OsO}_{4}\right) / 1.5 \%$ potassium ferrocyanide $\left(\mathrm{KFeCN}_{6}\right)$ for $1 \mathrm{~h}$, washed in water for three times and incubated in $1 \%$ aqueous uranyl acetate for $1 \mathrm{~h}$ followed by two washes in water and subsequent dehydration in grades of alcohol (10 min each; $50 \%, 70 \%$, $90 \%, 2 \times 10 \mathrm{~min} 100 \%)$. The samples were incubated in a $1: 1$ mixture of $100 \%$ ethanol and LR White (EMS), followed by two changes of pure LR White for $1 \mathrm{~h}$ followed by overnight incubation in pure LR white. The following day, the samples were placed in a new LR White resin in gelatin capsules (EMS) and polymerized at $60^{\circ} \mathrm{C}$ for $48 \mathrm{~h}$. Ultra-thin sections (about $80 \mathrm{~nm}$ ) were cut on a Reichert Ultracut-S microtome, picked up on copper grids, stained with lead citrate and examined in a JEOL 1200EX Transmission electron microscope. Images were recorded with an AMT $2 \mathrm{k}$ CCD camera and saved as TIFF files.

BBB opening using hypertonic solution. Hypertonic solution was generated by mixing $20 \%$ Mannitol Injection USP $\left(1100 \mathrm{mOsmol} \mathrm{L}^{-1}\right)$ and EC $\left(280 \mathrm{mOsmol} \mathrm{L}^{-1}\right)$ media in 1:3 ratio considering the dilution after intravenous or intra-arterial injection in the body. The isotonic solution was generated by mixing PBS and EC media in 1:3 ratio. The apparent permeability $\left(P_{\text {app }}\right)$ of fluorescently labeled $10 \mathrm{kDa}$ dextran tracer (Thermo Fisher) and cetuximab (Selleckchem.com, USA) on BBB Chip was measured before treating hypertonic solution and rinsed with regular EC media. To open the barrier, the endothelial channel in the BBB Chip was fed with hypertonic solution at $100 \mu \mathrm{h}^{-1}$ flow rate for $1 \mathrm{~h}(-1$ to $0 \mathrm{~h})$, and the media was switched to hypertonic solution including $100 \mu \mathrm{g} \mathrm{ml}^{-1}$ of $10 \mathrm{kDa}$ dextran and $10 \mu \mathrm{g} \mathrm{ml}^{-1}$ of cetuximab $(0-1$ h). After collecting the effluents at $1 \mathrm{~h}$, the media was switched to isotonic media with same dose of $10 \mathrm{kDa}$ dextran and cetuximab to let the barrier recover. The effluent was collected at $30 \mathrm{~min}, 1.5 \mathrm{~h}$, and $3.5 \mathrm{~h}$ after switching to isotonic media, and the $P_{\text {app }}$ of the $10 \mathrm{kDa}$ dextran and cetuximab were analyzed by microplate reader and human IgG1 ELISA kit (Sigma).

Metabolic barrier function. P-gp, MRP, and BCRP functionality were assessed using rhodamine 123 (Sigma), DiOC2 (3,3'-diethyloxacarbocyanine Iodide) (Sigma), citalopram (Sigma), and doxorubicin (Sigma). Both channels were pretreated with $50 \mu \mathrm{M}$ verapamil (Sigma), $10 \mu \mathrm{M}$ MK571 (Sigma), or $1 \mu \mathrm{M}$ Ko143, which are inhibitors of P-gp, MRPs, or BCRP, respectively. At $30 \mathrm{~min}$ after pretreatment with inhibitors, rhodamine $123(2 \mu \mathrm{M})$, DiOC2 $(2 \mu \mathrm{M})$, citalopram or doxorubicin $(5 \mu \mathrm{M})$ in the presence or absence of inhibitor was dosed to brain endothelial channel with flow rate at $100 \mu \mathrm{h} \mathrm{h}^{-1}$. To monitor the barrier integrity, $100 \mu \mathrm{g} \mathrm{ml}^{-1}$ of $3 \mathrm{kDa}$ dextran-cascade blue (Thermo Fisher) was dosed simultaneously. We collected apical and basal effluent for $6 \mathrm{~h}$ and tracers in the effluents were quantified by measuring fluorescent intensity. The fluorescence was measured at $485 / 530 \mathrm{~nm}, 482 / 497 \mathrm{~nm}$, or $470 / 585 \mathrm{~nm}$ to quantify rhodamine 123 , DiOC2, or doxorubicin, respectively, using a Synergy H1 microplate reader (BioTek, USA). Amount of citalopram in the apical and basal media was quantified using mass spectroscopy. The increase of $\mathrm{BBB}$ permeability of the drugs in the presence of inhibitor was presented as 'ratio of $P_{\text {app }}$ '.

BBB transcytosis of antibodies and peptide-modified Q-dots. For the transcytosis assay, $10 \mu \mathrm{g} \mathrm{ml}^{-1}$ of MEM-75 or $13 \mathrm{E} 4$ from Abcam (Cambridge, UK) was flowed to the brain endothelial channel at $60 \mu \mathrm{h} \mathrm{h}^{-1}$ for $3 \mathrm{~h}$, and the effluents were collected for the analysis. The detection and quantification of antibodies were performed by ELISA as described in previous report ${ }^{61}$. Briefly, $5 \mu \mathrm{gLL}^{-1}$ of goatanti-mouse antibody (Jackson ImmunoResearch) was immobilized on a high binding 96 -well plate in carbonate buffer $\mathrm{pH} 9.5$ for overnight at $4{ }^{\circ} \mathrm{C}$. Plate was washed with PBS with $0.1 \%$ Tween 20 for three times. $1 \%$ BSA in PBS was used to block the plate for $1 \mathrm{~h}$ at RT. Antibody samples collected from BBB Chips were incubated on the plate in the EC media for $2 \mathrm{~h}$ at RT. Then, an HRP-conjugated anti-mouse secondary antibody (Jackson ImmunoResearch) was used to detect the MEM75 and 13E4 antibodies on the plate. HRP enzyme on the secondary antibody was quantified using a luminescent substrate (Sigma). Standard curves for MEM75 and $13 \mathrm{E} 4$ antibodies was generated on the same plate to calculate the concentrations of the antibodies in the samples (Supplementary Figure 13). The luminescent intensity of the plates was measured using a Synergy H1 microplate reader (BioTek, USA), and the antibody concentrations in the samples from the brain and vascular channels of the BBB Chip was quantified to calculate the $P_{\text {app }}\left(\mathrm{cm} \mathrm{s}^{-1}\right)$.

To generate the Angiopep-2 modified Q-dot, C-terminally biotinylated Angiopep-2 (TFFYGGSRGKRNNFKTEEY) and scrambled Angiopep-2 (TGFKYRFGSKEGRNNYTEF) were purchased through the custom peptide synthesis service of Peptide 2.0 (USA), and Qdot 655 ITK Streptavidin Conjugate Kit was purchased from Thermo Fisher Scientific. Four micrometers of Cterminally biotinylated synthetic Angiopep-2 or scrambled Angiopep-2 was incubated with $0.4 \mu \mathrm{M}$ of Q-dots for $30 \mathrm{~min}$ at $4^{\circ} \mathrm{C}$ with agitation. The unbound peptides were cleared by using protein desalting column, 7K MWCO (Pierce) following instruction. Four micrometers of Angiopep-2 modified or scrambled Angiopep-2 modified Q-dot was dosed to brain endothelial channel at $100 \mu \mathrm{h} \mathrm{h}^{-1}$ for $6 \mathrm{~h}$, and the effluents were collected for analysis. The fluorescent intensity of effluent was measured using a Synergy H1 microplate reader (BioTek, USA) at 488/ $655 \mathrm{~nm}$, and the Q-dot was quantified to calculate the $P_{\text {app }}\left(\mathrm{cm} \mathrm{s}^{-1}\right)$.
Statistical analyses. All data represent means $( \pm$ SE) of distinct sample measurements $(N>2)$. The statistical analysis is two-way ANOVA with Tukey's multiple comparisons test; ${ }^{*} P<0.05 ;{ }^{* *} P<0.001 ;{ }^{* *} P<0.0001$. Prism 7 (GraphPad Software) was used for statistical analysis.

Reporting summary. Further information on research design is available in the Nature Research Reporting Summary linked to this article.

\section{Data availability}

All data generated or analyzed during this study are included in this published article (and its supplementary information files), or are available from the corresponding author upon request.

Received: 16 November 2018 Accepted: 16 May 2019 Published online: 13 June 2019

\section{References}

1. Cecchelli, R. et al. Modelling of the blood-brain barrier in drug discovery and development. Nat. Rev. Drug Discov. 6, 650-661 (2007).

2. Greene, C. \& Campbell, M. Tight junction modulation of the blood brain barrier: CNS delivery of small molecules. Tissue Barriers 4, el138017 (2016).

3. Mahringer, A., Ott, M., Reimold, I., Reichel, V. \& Fricker, G. The ABC of the blood-brain barrier-regulation of drug efflux pumps. Curr. Pharm. Des. 17, 2762-2770 (2011)

4. Shawahna, R. et al. Transcriptomic and quantitative proteomic analysis of transporters and drug metabolizing enzymes in freshly isolated human brain microvessels. Mol. Pharm. 8, 1332-1341 (2011).

5. Abbott, N. J. \& Friedman, A. Overview and introduction: the blood-brain barrier in health and disease. Epilepsia 53, 1-6 (2012).

6. Herland, A. et al. Distinct contributions of astrocytes and pericytes to neuroinflammation identified in a 3D human blood-brain barrier on a chip. PLoS One 11, e0150360 (2016).

7. Wolff, A., Antfolk, M., Brodin, B. \& Tenje, M. In vitro blood-brain barrier models-an overview of established models and new microfluidic approaches. J. Pharm. Sci. 104, 2727-2746 (2015).

8. Helms, H. C. et al. In vitro models of the blood-brain barrier: an overview of commonly used brain endothelial cell culture models and guidelines for their use. J. Cereb. Blood Flow. Metab. 36, 862-890 (2016).

9. Mason, W. P. Blood-brain barrier-associated efflux transporters: a significant but underappreciated obstacle to drug development in glioblastoma. NeuroOncol. 17, 1181-1182 (2015).

10. O'Brien, F. E., Dinan, T. G., Griffin, B. T. \& Cryan, J. F. Interactions between antidepressants and P-glycoprotein at the blood-brain barrier: clinical significance of in vitro and in vivo findings. Br. J. Pharmacol. 165, 289-312 (2012).

11. Jorfi, M., D'Avanzo, C., Kim, D. Y. \& Irimia, D. Three-dimensional models of the human brain development and diseases. Adv. Healthc. Mater. 7, 1700723 (2018).

12. Lauschke, K., Frederiksen, L. \& Hall, V. J. Paving the way toward complex blood-brain barrier models using pluripotent stem cells. Stem Cells Dev. 26, 857-874 (2017)

13. Lippmann, E. S. et al. Derivation of blood-brain barrier endothelial cells from human pluripotent stem cells. Nat. Biotechnol. 30, 783-791 (2012).

14. Sances, S. et al. Human iPSC-derived endothelial cells and microengineered organ-chip enhance neuronal development. Stem Cell Rep. 10, 1222-1236 (2018)

15. Gastfriend, B. D., Palecek, S. P. \& Shusta, E. V. Modeling the blood-brain barrier: beyond the endothelial cells. Curr. Opin. Biomed. Eng. 5 6-12 (2018)

16. Delsing, L. et al. Barrier properties and transcriptome expression in human iPSC-derived models of the blood-brain barrier. Stem Cells 36, 1816-1827 (2018)

17. Lippmann, E. S., Al-Ahmad, A., Azarin, S. M., Palecek, S. P. \& Shusta, E. V. A retinoic acid-enhanced, multicellular human blood-brain barrier model derived from stem cell sources. Sci. Rep. 4, 4160 (2014)

18. Vatine, G. D. et al. Modeling psychomotor retardation using iPSCs from MCT8-deficient patients indicates a prominent role for the blood-brain barrier. Cell Stem Cell 20, 831-843.e5 (2017).

19. Bhatia, S. N. \& Ingber, D. E. Microfluidic organs-on-chips. Nat. Biotechnol. 32, 760-772 (2014).

20. Kusuma, S., Peijnenburg, E., Patel, P. \& Gerecht, S. Low oxygen tension enhances endothelial fate of human pluripotent stem cells. Arterioscler. Thromb. Vasc. Biol. 34, 913-920 (2014). 
21. Lee, S. W. et al. Hypoxic priming of mESCs accelerates vascular-lineage differentiation through HIF1-mediated inverse regulation of Oct4 and VEGF. EMBO Mol. Med. 4, 924-938 (2012).

22. Han, Y., Kuang, S.-Z., Gomer, A. \& Ramirez-Bergeron, D. L. Hypoxia influences the vascular expansion and differentiation of embryonic stem cell cultures through the temporal expression of vascular endothelial growth factor receptors in an ARNT-dependent manner. Stem Cells 28, 799-809 (2010).

23. Abdollahi, H. et al. The role of hypoxia in stem cell differentiation and therapeutics. J. Surg. Res.165, 112-117 (2011).

24. Katt, M. E., Xu, Z. S., Gerecht, S. \& Searson, P. C. Human brain microvascular endothelial cells derived from the BC1 iPS cell line exhibit a blood-brain barrier phenotype. PLoS One 11, e0152105 (2016).

25. Stenman, J. M. et al. Canonical Wnt signaling regulates organ-specific assembly and differentiation of CNS vasculature. Science 322, 1247-1250 (2008).

26. Daneman, R. et al. Wnt/ $\beta$-catenin signaling is required for $\mathrm{CNS}$, but not non-CNS, angiogenesis. Proc. Natl Acad. Sci. USA 106, 641-646 (2009).

27. Zhang, Q. et al. Wnt/ $\beta$-catenin signaling enhances hypoxia-induced epithelialmesenchymal transition in hepatocellular carcinoma via crosstalk with hif- $1 \alpha$ signaling. Carcinogenesis 34, 962-973 (2013).

28. $\mathrm{Xu}, \mathrm{W}$. et al. Hypoxia activates $\mathrm{Wnt} / \beta$-catenin signaling by regulating the expression of BCL9 in human hepatocellular carcinoma. Sci. Rep. 7, 40446 (2017).

29. Kim, K. S., Rajagopal, V., Gonsalves, C., Johnson, C. \& Kalra, V. K. A novel role of hypoxia-inducible factor in cobalt chloride- and hypoxia-mediated expression of IL-8 chemokine in human endothelial cells. J. Immunol. 177, 7211-7224 (2006).

30. Geier, E. G. et al. Profiling solute carrier transporters in the human bloodbrain barrier. Clin. Pharmacol. Ther. 94, 636-639 (2013).

31. Baeten, K. M. \& Akassoglou, K. Extracellular matrix and matrix receptors in blood-brain barrier formation and stroke. Dev. Neurobiol. 71, 1018-1039 (2011).

32. Henry, O. Y. F. et al. Organs-on-chips with integrated electrodes for transepithelial electrical resistance (TEER) measurements of human epithelial barrier function. Lab Chip 17, 2264-2271 (2017).

33. Stebbins, M. J. et al. Differentiation and characterization of human pluripotent stem cell-derived brain microvascular endothelial cells. Methods 101, 93-102 (2016).

34. Chen, J. F. et al. A(2A) adenosine receptor deficiency attenuates brain injury induced by transient focal ischemia in mice. J. Neurosci. 19, 9192-9200 (1999).

35. Wang, Y. I., Abaci, H. E. \& Shuler, M. L. Microfluidic blood-brain barrier model provides in vivo-like barrier properties for drug permeability screening. Biotechnol. Bioeng. 114, 184-194 (2017).

36. Löscher, W. \& Potschka, H. Blood-brain barrier active efflux transporters: ATP-binding cassette gene family. NeuroRx 2, 86-98 (2005).

37. Kalvass, J. C. et al. Why clinical modulation of efflux transport at the human blood-brain barrier is unlikely: the ITC evidence-based position. Clin. Pharmacol. Ther. 94, 80-94 (2013).

38. Semenza, G. L. Hypoxia-inducible factors in physiology and medicine. Cell 148, 399-408 (2012).

39. Triantafyllou, A. et al. Cobalt induces hypoxia-inducible factor-1a (HIF-1a) in HeLa cells by an iron-independent, but ROS-, PI-3K- and MAPK-dependent mechanism. Free Radic. Res. 40, 847-856 (2006).

40. Armada, A. et al. in Methods in Molecular Biology Vol. 1395, 87-103 (Humana Press, New York, NY, 2016).

41. Doran, A. et al. The impact of P-glycoprotein on the disposition of drugs targeted for indications of the central nervous system: evaluation using the MDR1A/1B knockout mouse model. Drug Metab. Dispos. 33, 165-174 (2005).

42. Feng, B. et al. In vitro P-glycoprotein assays to predict the in vivo interactions of P-glycoprotein with drugs in the central nervous system. Drug Metab. Dispos. 36, 268-275 (2008).

43. Rochat, B., Baumann, P. \& Audus, K. L. Transport mechanisms for the antidepressant citalopram in brain microvessel endothelium. Brain Res. 831, 229-236 (1999).

44. Li, M. et al. Identification of interspecies difference in efflux transporters of hepatocytes from dog, rat, monkey and human. Eur. J. Pharm. Sci. 35, 114-126 (2008).

45. Wang, Q., Yang, H., Miller, D. W. \& Elmquist, W. F. Effect of the Pglycoprotein inhibitor, cyclosporine A, on the distribution of rhodamine-123 to the brain: an in vivo microdialysis study in freely moving rats. Biochem. Biophys. Res. Commun. 211, 719-726 (1995).

46. Weksler, B. B. et al. Blood-brain barrier-specific properties of a human adult brain endothelial cell line. FASEB J. 19, 1872-1874 (2005).
47. Hollmann, E. K. et al. Accelerated differentiation of human induced pluripotent stem cells to blood-brain barrier endothelial cells. Fluids Barriers CNS 14, 9 (2017).

48. Ohnishi, T. et al. In vivo and in vitro evidence for ATP-dependency of P-glycoprotein-mediated efflux of doxorubicin at the blood-brain barrier. Biochem. Pharmacol. 49, 1541-1544 (1995).

49. Cisternino, S., Rousselle, C., Lorico, A., Rappa, G. \& Scherrmann, J. M. Apparent lack of mrpl-mediated efflux at the luminal side of mouse bloodbrain barrier endothelial cells. Pharm. Res 20, 904-909 (2003).

50. Burger, H. et al. Imatinib mesylate (STI571) is a substrate for the breast cancer resistance protein (BCRP)/ABCG2 drug pump. Blood 104, 2940-2942 (2004)

51. Neuwelt, E. A., Minna, J., Frenkel, E., Barnett, P. A. \& McCormick, C. I. Osmotic blood-brain barrier opening to IgM monoclonal antibody in the rat. Am. J. Physiol. 250, R875-R883 (1986).

52. Chakraborty, S. et al. Superselective intraarterial cerebral infusion of cetuximab after osmotic blood/brain barrier disruption for recurrent malignant glioma: phase I study. J. Neurooncol. 128, 405-415 (2016).

53. Roman-Goldstein, $\mathrm{S}$. et al. MR and cognitive testing of patients undergoing osmotic blood-brain barrier disruption with intraarterial chemotherapy. Ajnr. Am. J. Neuroradiol. 16, 543-553 (1995).

54. Kiviniemi, V. et al. Real-time monitoring of human blood-brain barrier disruption. PLoS One 12, e0174072 (2017).

55. Pardridge, W. M. Blood-brain barrier drug delivery of IgG fusion proteins with a transferrin receptor monoclonal antibody. Expert Opin. Drug Deliv. 12, 207-222 (2015)

56. Oller-Salvia, B., Sánchez-Navarro, M., Giralt, E. \& Teixidó, M. Blood-brain barrier shuttle peptides: An emerging paradigm for brain delivery. Chem. Soc. Rev. 45, 4690-4707 (2016).

57. Demeule, M. et al. Involvement of the low-density lipoprotein receptor-related protein in the transcytosis of the brain delivery vector Angiopep-2. J. Neurochem 106, 1534-1544 (2008).

58. Thomas, F. C. et al. Uptake of ANG1005, a novel paclitaxel derivative, through the blood-brain barrier into brain and experimental brain metastases of breast cancer. Pharm. Res. 26, 2486-2494 (2009).

59. Regina, A. et al. ANG4043, a novel brain-penetrant peptide-mAb conjugate, is efficacious against HER2-positive intracranial tumors in mice. Mol. Cancer Ther. 14, 129-140 (2015).

60. Yu, Y. J. et al. Therapeutic bispecific antibodies cross the blood-brain barrier in nonhuman primates. Sci. Transl. Med 6, 261 ra154 (2014).

61. Sade, H. et al. A human blood-brain barrier transcytosis assay reveals antibody transcytosis influenced by $\mathrm{pH}$-dependent receptor binding. PLoS One 9, e96340 (2014)

62. Ribecco-Lutkiewicz, M. et al. A novel human induced pluripotent stem cell blood-brain barrier model: applicability to study antibody-triggered receptormediated transcytosis. Sci. Rep. 8, 1873 (2018).

63. Wevers, N. R. et al. A perfused human blood-brain barrier on-a-chip for highthroughput assessment of barrier function and antibody transport. Fluids Barriers CNS 15, 23 (2018).

64. Linville, R. M. et al. Human iPSC-derived blood-brain barrier microvessels: Validation of barrier function and endothelial cell behavior. Biomaterials 190-191, 24-37 (2018).

65. Campisi, M. et al. 3D self-organized microvascular model of the human blood-brain barrier with endothelial cells, pericytes and astrocytes. Biomaterials 180, 117-129 (2018).

66. Srinivasan, B. et al. TEER measurement techniques for in vitro barrier model systems. J. Lab. Autom. 20, 107-126 (2015).

67. Zhdanov, A. V., Okkelman, I. A., Collins, F. W. J., Melgar, S. \& Papkovsky, D. B. A novel effect of DMOG on cell metabolism: direct inhibition of mitochondrial function precedes HIF target gene expression. Biochim. Biophys. Acta - Bioenerg. 1847, 1254-1266 (2015).

68. Brown, J. A. et al. Recreating blood-brain barrier physiology and structure on chip: A novel neurovascular microfluidic bioreactor. Biomicrofluidics $\mathbf{9}$, 054124 (2015).

69. Katt, M. E., Linville, R. M., Mayo, L. N., Xu, Z. S. \& Searson, P. C. Functional brain-specific microvessels from iPSC-derived human brain microvascular endothelial cells: the role of matrix composition on monolayer formation. Fluids Barriers CNS 15, 7 (2018).

70. Cucullo, L., Hossain, M., Puvenna, V., Marchi, N. \& Janigro, D. The role of shear stress in Blood-Brain Barrier endothelial physiology. BMC Neurosci. 12, 40 (2011).

71. Yeh, W.-L., Lu, D.-Y., Lin, C.-J., Liou, H.-C. \& Fu, W.-M. Inhibition of hypoxia-induced increase of blood-brain barrier permeability by YC-1 through the antagonism of HIF-1 accumulation and VEGF expression. Mol. Pharmacol. 72, 440-449 (2007).

72. Doeppner, T. R. et al. The novel proteasome inhibitor BSc2118 protects against cerebral ischaemia through HIF1A accumulation and enhanced angioneurogenesis. Brain 135, 3282-3297 (2012). 
73. Maoz, B. M. et al. A linked organ-on-chip model of the human neurovascular unit reveals the metabolic coupling of endothelial and neuronal cells. Nat. Biotechnol. 36, 865-874 (2018).

74. Benam, K. H. et al. Small airway-on-a-chip enables analysis of human lung inflammation and drug responses in vitro. Nat. Methods 13, 151-157 (2016).

75. Tang, L. \& Lee, N. Y. A facile route for irreversible bonding of plastic-PDMS hybrid microdevices at room temperature. Lab Chip 10, 1274 (2010).

\section{Acknowledgements}

This research was supported by funding from the Wyss Institute for Biologically Inspired Engineering (to D.E.I.), Defense Advanced Research Projects Agency under Cooperative Agreement Number W911NF-12-2-0036 (to D.E.I), and National Research Foundation of Korea (NRF) grant (Korea, government, Ministry of Science and ICT; NRF2018R1A5A1024340 and 2018K1A4A3A01063890 to T-E.P.), and Knut and Alice Wallenberg Foundation (WAF 2015-0178) (to A.H.). We also thank T. Ferrante for technical assistance, and L. Jin for artwork and technical illustration.

\section{Author contributions}

T-E.P. and N.M. participated in the design and performance of all experiments and analyzed the data, working with D.E.I., who also supervised all work. R.H., R.M., E.H.F., and H.S. helped perform experiments. A.W. and R.P-B. helped design experiments, interpret data, and supervised all work. A.H. helped design experiments, performed imaging and proteomics experiments, and interpret data. O.H. and M.B. prepared microfluidic chips and helped measure TEER and interpret data. H.J.M. and L.C.G. provided scientific vision on the experiments related to reversible osmotic opening of the human BBB. H.W.S. and S.P.P. help develop protocols for the differentiation of stem cells. E.S. provided scientific supervision on the BBB model as well as access to training on the differentiation of stem cells to the brain endothelial cells. N.M., T-E.P., and D.E.I. prepared the paper with input from all others.

\section{Additional information}

Supplementary Information accompanies this paper at https://doi.org/10.1038/s41467019-10588-0.

Competing interests: D.E.I. is a founder and holds equity in Emulate, Inc., and chairs its scientific advisory board. All other authors declare no competing interests.

Reprints and permission information is available online at http://npg.nature.com/ reprintsandpermissions/

Journal peer review information: Nature Communications thanks the anonymous reviewer(s) for their contribution to the peer review of this work. Peer reviewer reports are available.

Publisher's note: Springer Nature remains neutral with regard to jurisdictional claims in published maps and institutional affiliations.

\section{(c) (i)}

Open Access This article is licensed under a Creative Commons Attribution 4.0 International License, which permits use, sharing, adaptation, distribution and reproduction in any medium or format, as long as you give appropriate credit to the original author(s) and the source, provide a link to the Creative Commons license, and indicate if changes were made. The images or other third party material in this article are included in the article's Creative Commons license, unless indicated otherwise in a credit line to the material. If material is not included in the article's Creative Commons license and your intended use is not permitted by statutory regulation or exceeds the permitted use, you will need to obtain permission directly from the copyright holder. To view a copy of this license, visit http://creativecommons.org/ licenses/by/4.0/.

(C) The Author(s) 2019 